\title{
Role of Ghrelin in Postmenopausal obesity
}

\author{
Mona Mohamed Ibrahim Abdalla ${ }^{1,2^{*}}$, Ravindran Jegasothy ${ }^{2}$
}

\begin{abstract}
Objectives: Obesity is considered a worldwide important health problem with continuously increasing prevalence, particularly among postmenopausal women. Weight gain is one of the major concerns of menopause. Understanding the contributing factors to postmenopausal obesity helps to reduce its incidence and thus its associated health complications. The present study aimed to review the role of ghrelin in mediating postmenopausal obesity.

Methods: A literature review was done to understand the possible association between ghrelin levels and obesity in postmenopausal women.

Results: It was found that the lack of estrogen during menopause increases the serum ghrelin level. In addition, the results revealed that ghrelin stimulates appetite, changes food preference, and causes an accumulation of visceral fat resulting in weight gain. On the other hand, ghrelin was found to have many protective effects as an anti-inflammatory and a neuroprotective agent.

Conclusions: In general, the ghrelin hormone is a double-edged sword as it mediates the increase of body weight in postmenopausal women. More importantly, it plays a considerable role in protecting postmenopausal women from cardiovascular and neurodegenerative diseases. The selective blockage of appetite-stimulating and metabolic effects of ghrelin may be of a potential prophylactic or therapeutic effect for postmenopausal overweight and obesity.

Keywords: Postmenopausal obesity, Ghrelin, Satiation, Estrogen, Adipokines
\end{abstract}

\section{The Burden of Postmenopausal Obesity and its Contributing Factors}

The incidence of overweight, as well as obesity is increasing worldwide. Based on the World Health Organization (WHO) report, obesity prevalence nearly tripled between 1975 and 2016 with a high prevalence among women where 39\% of women aged 18 were overweight in 2016. Further, the WHO defines overweight and/or obesity as "an abnormal or excessive fat accumulation that may impair health". Obesity is also described as "a chronic disease that is characterized by the excessive accumulation of fat tissues and increases the risk of so-called civilization diseases such as cardiovascular and metabolic disorders, as well as cancers" (1).

Women are at higher risk of overweight or obese, particularly as they grow older and postmenopausal women are a particularly vulnerable population for obesity and its consequences (2). In the United States, $66.3 \%$ of women aged 40-59 years were found to be overweight and obese with a prevalence rate of around $38.2 \%$ (2). The Family Budget Study data in Brazil revealed that $58.0 \%$ and $63.0 \%$ of women aged 45-54 and 55-64 were overweight, out of these, $21.5 \%$ and $26.0 \%$ were obese, respectively (3). The increasing prevalence of obesity is directly correlated with an increase in morbidity and mortality rates (4).

Menopause marks the cessation of menstrual cycles and is defined as "happening 12 months after the final menstrual period". It is caused by the depletion of the gonadal follicles and a decrease in estrogen secretion. Premenopause is known as a period of quite regular menses that precedes menopause, which refers to the duration of 3-12 months with irregular bleeding preceding menopause. The increased incidence of weight gain among postmenopausal women is also accompanied with a high incidence of several diseases such as diabetes, metabolic syndrome, cardiovascular diseases, and even cancer $(5,6)$.

In addition to the genetic predisposition, many other factors contribute to the increase in body fat accumulation in postmenopausal women (7). Contributory factors include the ethnic group, reduced lean body mass, diminished resting metabolism, and the use of certain medications such as steroids, glitazones, and insulin (7), as well as the lack of estrogen and reduced physical activity as the main reasons of this condition (8). The reduction in the estrogen level during menopause, along with its associated alterations in fat metabolism and deposits plays a fundamental role in the prevalence of such health problems among postmenopausal women. In conditions of hypoestrogenemia, the fat tends to deposit around visceral organs $(9,10)$.

The adipose tissue is classified into white and brown fatty tissues. The white adipose is divided into subcutaneous fat, which is accumulated below the skin, and visceral fat that surrounds the internal organs such

Received 24 March 2019, Accepted 7 August 2019, Available online 10 September 2019

${ }^{1}$ Human Biology Department, School of Medicine, International Medical University, Malaysia. ${ }^{2}$ Faculty of Medicine, MAHSA University, Malaysia. *Corresponding Author: Mona Mohamed Ibrahim Abdalla, Tel: +601111362625, Fax: +6017.3522107, Email: monaalbetar@yahoo.com 
as kidney, liver, and intestine. Adipocytes, as the main cells of the adipose tissue, undergo hypertrophy and hyperplasia in response to increased triacylglycerol storage and this leads to an alteration in their metabolic activities. In addition, adipocytes secrete substances known as "adipokines such as leptin and adiponectin" that play a role in insulin sensitivity and the regulation of food intake and subsequently body weight. Recent studies have shown a strong correlation between increased incidences of diseases with obesity, particularly visceral obesity (11).

The maintenance of body weight relies on balancing energy input with energy expenditure. Further, energy homeostasis is controlled by many central and peripheral peptides such as ghrelin, leptin, adiponectin, and insulin. The alteration of one of these peptides or more during menopause may contribute to a change in body weight and its composition (12). In addition to the role of the alterations of these peptides in causing obesity, they are claimed to be the reason behind the difficulty in weight reduction, as well as the maintenance of this reduced weight among postmenopausal women even with intensive behavioral weight loss programs (13). Furthermore, the alteration of these adipokines contributes to the development of postmenopausal obesity-associated conditions such as insulin resistance, coronary heart diseases, and impaired cognitive function $(14,15)$.

\section{Ghrelin Hormone in Postmenopausal Women}

Ghrelin, as a growth hormone secretagogue (GHS) peptide, is chemically formed of 28-amino acids and is the only known appetite-stimulating or orexigenic peptide (16). The gastric oxyntic cells are considered as the main ghrelin secreting cells (17) that are characterized by their close contact with the capillaries, suggesting the endocrine functions of ghrelin (18). Ghrelin is also synthesized in small quantities in the rest of the gastrointestinal tract by another kind of ghrelin-producing cells which lies in close contact with the lumen of the intestine (19). These two different kinds of cells may play distinctive physiological functions. In addition, ghrelin manufacturing is attributed to placenta, pancreas, kidney, pituitary, and hypothalamus (20).

Circulating ghrelin has unacylated and acylated forms. The acylated form is also known as "ghrelin or AG" and represents $10 \%$ of the whole circulating ghrelin (21). The actions of ghrelin are mediated by two subtypes of G-protein-coupled receptors, type 1a and $1 b$ GHS receptors (Rs), which are found in the pituitary, hypothalamus, kidney, or adipose tissues (21).

In addition to its effect on appetite and body weight, ghrelin plays an essential role in glucose homeostasis. It also stimulates the motility and secretion of the hydrochloric acid by the stomach and inhibits the secretion of pepsin (21). Further, the serum level of ghrelin increases during starvation, weight loss, and upon the increase in blood insulin levels while it decreases during the period of satiety after meals, especially fat and carbohydrate-rich meals (22).

The unacylated form of ghrelin that is also referred to as "des-acyl ghrelin or DAG" demonstrates the dominant plasma form (90\%). It was thought earlier to be an inactive form of the ghrelin because it could not bind to GHSR1a, the type of ghrelin receptors that mediates the biological actions of the ghrelin (16). Later, it was found that DAG plays a crucial role in many physiological functions and pathological conditions (23). It is considered as a separate hormone that acts independently or together with AG (24). Obese subjects exhibited an increase in their DAG plasma levels associated with unchanged AG levels, indicating the role of body weight in the regulation of DAG (25). Moreover, DAG promotes the loss of body weight by reducing fat mass and AG. It also enhances glucose utilization by increasing postprandial insulin release and insulin sensitivity (26) The association between the effect of DAG on the postprandial glucose level and the pre-prandial levels of AG reflects the regulatory role of DAG in individuals with relatively increased AG levels (24). It should be noted that DAG effects are not blocked by GHSR1a-blockers contrary to the effectiveness of such blockers on inhibiting AG-mediated insulin release $(27,28)$.

There are conflicting findings regarding the role of ovarian hormones in regulating ghrelin secretion. Thus, "estradiol exerts a tonic inhibitory effect on ghrelin release in females" as evidenced by the increase of food intake, body weight, plasma ghrelin level, hypothalamic neuropeptide $\mathrm{Y}$, as well as agouti-related protein mRNA expressions that were reported after ovariectomy (OVX), an experimentally induced menopause (29). It was suggested that the increased food intake following OVX is because of "releasing ghrelin secretion from the tonic inhibitory effect of estradiol". These data suggest that "estradiol inhibits the orexigenic action of ghrelin in females and that weight gain associated with OVX is ghrelin-mediated" (29). This drives the attention toward the possible role of ghrelin in mediating postmenopausal obesity and the potential effectiveness of ghrelin antagonists as a treatment of postmenopausal obesity. Contrarily, it was shown that short-term exogenous estrogen did not modify the ghrelin level in neither premenopausal nor post-menopausal women, suggesting that "ovarian hormones are not involved in the regulation of ghrelin secretion in women" (30).

The enhanced appetite and visceral fat accumulation in postmenopausal women may be caused by an increase in the ghrelin level which may also explain the difficulty that postmenopausal women face in maintaining weight loss (Figure 1). Obesity-associated complications, especially visceral obesity is linked to an increase in the levels of some inflammatory markers, along with an alteration in adipokines. A study conducted by St-Pierre et al on the association between the serum ghrelin level in 


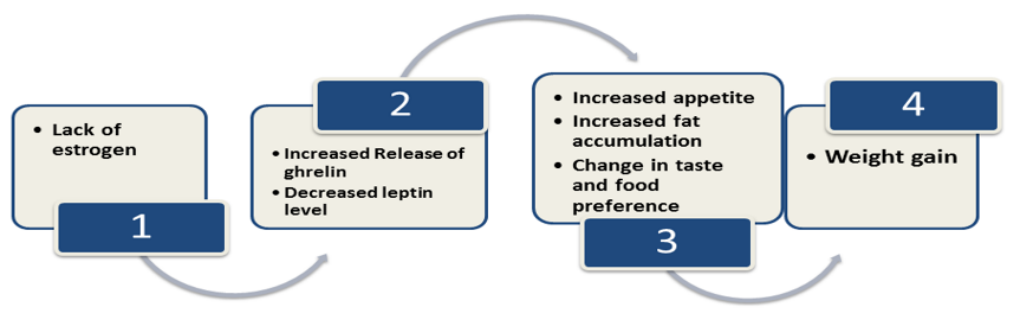

Figure 1. Ghrelin as a Mediator for Postmenopausal Obesity.

overweight and obese postmenopausal women with some inflammatory markers such as tumor necrosis factor-alpha (TNF-alpha) showed a positive association between AG and TNF-alpha (31), reflecting the role of a high ghrelin level in overweight and obese postmenopausal women in mediating the associated complications.

However, conflicting reports exist on the impact of visceral fat versus subcutaneous body fat on the ghrelin level. Ikezaki et al reported a negative association between visceral fat and the ghrelin level, explaining that higher insulin resistance which accompanies visceral fat accumulation results in the inhibition of ghrelin secretion (32). This was contradictory to the results reported by Sondergaard et al on the visceral fat as a strong predictor of the serum ghrelin levels in premenopausal women. Based on their results, higher levels of visceral fat led to higher ghrelin levels (33). These studies indicate that the correlation between the ghrelin level and the visceral fat differs in different premenopausal women, which could be due to the hormonal changes associated with the transition from pre- to postmenopause.

It seems that an increase in the ghrelin level in both overweight and obese postmenopausal women represents a physiological response as evidenced by the reported beneficial effects of ghrelin such as being a vasodilator (34) and having anti-inflammatory properties (34-36). The lower concentration of ghrelin was found to be associated with carotid atherosclerosis (37) and stroke (38). For instance, Karim et al concluded that "postmenopausal women greater than 10 years into menopause had significantly lower concentrations of ghrelin compared with women within 6 years of menopause, which can partially explain the increased risk of stroke and cardiovascular diseases in this population among older postmenopausal women" (39).

Similarly, Isomoto et al found the anti-inflammatory effect of ghrelin and reported the gastroprotective effect of the increased ghrelin level in acute gastritis, a response which is associated with an occurring inflammatory process (40). Ghrelin causes an increase in the local blood flow and the production of prostaglandins $(20,40,41)$. These findings may explain the higher ghrelin concentrations in lean individuals and patients with chronic liver disease, celiac disease, bulimia, or anorexia nervosa $(42,43)$. The use of combined oral contraceptives increases the concentration of ghrelin in women with severe malnutrition (44). Conversely, lowering the concentration of ghrelin is linked to obesity and insulin resistance (45). In addition, ghrelin was found to show a neuroprotective effect on mitochondrial respiration through its stimulatory effect. In higher brain functions, ghrelin takes part in the regulation of the "sleep-wake state" (46), as well as "learning and memory consolidation" (47). All these beneficial actions of ghrelin make it very important for aging women and attract attention to its potential therapeutic effects for many neurodegenerative diseases that are common in old individuals including postmenopausal women (48). The change in the ghrelin level during the stage of perimenopause was also found to be associated with the alterations of other adipokines such as adiponectin as compared to pre- and postmenopause stages (49).

In general, the findings of previous studies on the ghrelin level in and its effects on postmenopausal women indicate that the increase of ghrelin hormone after menopause is a physiological response to the post-menopausalinflammatory response. Further, ghrelin acts as an antiinflammatory hormone and provides many protective actions although it simultaneously increases food intake and fat accumulation leading to more inflammation. An increase in body weight to a certain level or maybe through a negative feedback mechanism leads to a reduction in the ghrelin level with the advance of postmenopause during the following years. This could cause the worsening of the associated inflammation and explain the increase of the risk for different diseases. Accordingly, the selective inhibition of the orexigenic effect of ghrelin or the prolongation of the satiety time may be of value in treating postmenopausal obesity.

\section{Changes in Satiety in Postmenopausal Women and the Role of Ghrelin}

Satiety is the time interval between the meals with no hunger. The termination of this interval coincides with the feeling of the hunger and intake of the next meal that is correlated with the increase of ghrelin and the decrease of leptin, which is considered as the satiety hormone (50). The alterations in ghrelin and leptin levels with menopause are also related to the changes in olfaction and gustation which play a great influence on food preference 
and feeding behaviour (49). This was evidenced by the enhanced expression of ghrelin and its receptors reported in type I, II, and III taste bud cells. The response to the citric acid and sodium chloride was lower in the GHS-R null mice as compared with wild-type controls (51). A recent study investigated the olfaction and taste functions in obese female patients and their association with serum ghrelin and leptin levels compared with healthy controls. Based on the reports, an inverse relationship was found between the serum level of leptin with olfaction and taste while there was an insignificant correlation between the ghrelin level and the alterations in both sensations (52).

\section{Conclusions}

Overall, ghrelin is a major peptide for food intake stimulation in addition to its positive energy balancepromoting effect and weight gain. It is suggested that antagonizing the effects of ghrelin by receptor antagonists blocks the transmission of orexigenic signals from the gastrointestinal tract to the brain and, subsequently, may be used as effective anti-obesity therapies. As a result, the developmentofghrelinantagonistsisanarea ofinvestigation on the part of pharmaceutical companies. Thus, it will be of great interest to investigate the effectiveness of interventions that aim to decrease the circulating ghrelin levels or targeting its receptor antagonists in treating the weight gain of the postmenopause, as well as its associated complications.

\section{Conflict of Interests}

Authors declare that they have no conflict of interests.

\section{Ethical Issues}

Not applicable.

\section{Financial Support}

The publication of this article was supported by MAHSA University, Malaysia.

\section{References}

1. Obesity and overweight: key facts. WHO website. http:// www.who.int/news-room/fact-sheets/detail/obesity-andoverweight.

2. Flegal KM, Carroll MD, Ogden CL, Curtin LR. Prevalence and trends in obesity among US adults, 1999-2008. JAMA. 2010;303(3):235-241. doi:10.1001/jama.2009.2014

3. Gravena AA, Brischiliari SC, Lopes TC, Agnolo CM, Carvalho MD, Pelloso SM. Excess weight and abdominal obesity in postmenopausal Brazilian women: a populationbased study. BMC Womens Health. 2013;13:46. doi:10.1186/1472-6874-13-46

4. Abdelaal M, le Roux CW, Docherty NG. Morbidity and mortality associated with obesity. Ann Transl Med. 2017;5(7):161. doi:10.21037/atm.2017.03.107

5. Lambrinoudaki I, Brincat $M$, Erel CT, et al. EMAS position statement: managing obese postmenopausal women. Maturitas. 2010;66(3):323-326. doi:10.1016/j. maturitas.2010.03.025

6. Zheng H, Tumin D, Qian Z. Obesity and mortality risk: new findings from body mass index trajectories. Am J Epidemiol. 2013;178(11):1591-1599. doi:10.1093/aje/kwt179

7. Neuhouser ML, Aragaki AK, Prentice RL, et al. Overweight, obesity, and postmenopausal invasive breast cancer risk: a secondary analysis of the women's health initiative randomized clinical trials. JAMA Oncol. 2015;1(5):611621. doi:10.1001/jamaoncol.2015.1546

8. Kozakowski J, Gietka-Czernel M, Leszczyńska D, Majos A. Obesity in menopause - our negligence or an unfortunate inevitability? Prz Menopauzalny. 2017;16(2):61-65. doi:10.5114/pm.2017.68594

9. Dubnov-Raz G, Pines A, Berry EM. Diet and lifestyle in managing postmenopausal obesity. Climacteric. 2007;10 Suppl 2:38-41. doi:10.1080/13697130701586428

10. Pedersen SB, Kristensen K, Hermann PA, Katzenellenbogen JA, Richelsen B. Estrogen controls lipolysis by up-regulating alpha2A-adrenergic receptors directly in human adipose tissue through the estrogen receptor alpha. Implications for the female fat distribution. J Clin Endocrinol Metab. 2004;89(4):1869-1878. doi:10.1210/jc.2003-031327

11. Foryst-Ludwig A, Kintscher U. Metabolic impact of estrogen signalling through ERalpha and ERbeta. J Steroid Biochem Mol Biol. 2010;122(1-3):74-81. doi:10.1016/j. jsbmb.2010.06.012

12. Janochova K, Haluzik M, Buzga M. Visceral fat and insulin resistance - what we know? Biomed Pap Med Fac Univ Palacky Olomouc Czech Repub. 2019;163(1):19-27. doi:10.5507/bp.2018.062

13. Soni AC, Conroy MB, Mackey RH, Kuller LH. Ghrelin, leptin, adiponectin, and insulin levels and concurrent and future weight change in overweight, postmenopausal women. Menopause. 2011;18(3):296-301. doi:10.1097/ gme.0b013e3181f2e611

14. De Franciscis P, Barbieri M, Leo S, et al. Serum adiponectin levels are associated with worse cognitive function in postmenopausal women. PLoS One. 2017;12(12):e0186205. doi:10.1371/journal.pone.0186205

15. Kuller LH, Kinzel LS, Pettee KK, et al. Lifestyle intervention and coronary heart disease risk factor changes over 18 months in postmenopausal women: the Women On the Move through Activity and Nutrition (WOMAN study) clinical trial. J Womens Health (Larchmt). 2006;15(8):962974. doi:10.1089/jwh.2006.15.962

16. Kojima M, Hosoda H, Date Y, Nakazato M, Matsuo H, Kangawa K. Ghrelin is a growth-hormone-releasing acylated peptide from stomach. Nature. 1999;402(6762):656-660. doi:10.1038/45230

17. Date $Y$, Kojima M, Hosoda H, et al. Ghrelin, a novel growth hormone-releasing acylated peptide, is synthesized in a distinct endocrine cell type in the gastrointestinal tracts of rats and humans. Endocrinology. 2000;141(11):4255-4261. doi:10.1210/endo.141.11.7757

18. Camiña JP, Carreira MC, El Messari S, Llorens-Cortes C, Smith RG, Casanueva FF. Desensitization and endocytosis mechanisms of ghrelin-activated growth hormone secretagogue receptor 1a. Endocrinology. 2004;145(2):930940. doi:10.1210/en.2003-0974

19. Sakata I, Nakamura K, Yamazaki M, et al. Ghrelinproducing cells exist as two types of cells, closed- and 
opened-type cells, in the rat gastrointestinal tract. Peptides. 2002;23(3):531-536. doi:10.1016/s0196-9781(01)00633-7

20. Meier U, Gressner AM. Endocrine regulation of energy metabolism: review of pathobiochemical and clinical chemical aspects of leptin, ghrelin, adiponectin, and resistin. Clin Chem. 2004;50(9):1511-1525. doi:10.1373/ clinchem.2004.032482

21. Hosoda H, Kojima M, Matsuo H, Kangawa K. Ghrelin and des-acyl ghrelin: two major forms of rat ghrelin peptide in gastrointestinal tissue. Biochem Biophys Res Commun. 2000;279(3):909-913. doi:10.1006/bbrc.2000.4039

22. Konturek SJ, Konturek JW, Pawlik T, Brzozowski T. Braingut axis and its role in the control of food intake. J Physiol Pharmacol. 2004;55(1 Pt 2):137-154.

23. Weigle DS, Cummings DE, Newby PD, et al. Roles of leptin and ghrelin in the loss of body weight caused by a low fat, high carbohydrate diet. J Clin Endocrinol Metab. 2003;88(4):1577-1586. doi:10.1210/jc.2002-021262

24. Thompson NM, Gill DA, Davies R, et al. Ghrelin and desoctanoyl ghrelin promote adipogenesis directly in vivo by a mechanism independent of the type 1a growth hormone secretagogue receptor. Endocrinology. 2004;145(1):234242. doi:10.1210/en.2003-0899

25. Delhanty PJ, Huisman M, Julien M, et al. The acylated (AG) to unacylated (UAG) ghrelin ratio in esterase inhibitortreated blood is higher than previously described. Clin Endocrinol (Oxf). 2015;82(1):142-146. doi:10.1111/ cen.12489

26. Pacifico L, Poggiogalle E, Costantino F, et al. Acylated and nonacylated ghrelin levels and their associations with insulin resistance in obese and normal weight children with metabolic syndrome. Eur J Endocrinol. 2009;161(6):861870. doi:10.1530/eje-09-0375

27. Benso A, St-Pierre DH, Prodam F, et al. Metabolic effects of overnight continuous infusion of unacylated ghrelin in humans. Eur J Endocrinol. 2012;166(5):911-916. doi:10.1530/eje-11-0982

28. Gauna C, Delhanty PJ, van Aken MO, et al. Unacylated ghrelin is active on the INS-1E rat insulinoma cell line independently of the growth hormone secretagogue receptor type 1a and the corticotropin releasing factor 2 receptor. Mol Cell Endocrinol. 2006;251(1-2):103-111. doi:10.1016/j.mce.2006.03.040

29. Clegg DJ, Brown LM, Zigman JM, et al. Estradiol-dependent decrease in the orexigenic potency of ghrelin in female rats. Diabetes. 2007;56(4):1051-1058. doi:10.2337/db06-0015

30. Dafopoulos K, Chalvatzas N, Kosmas G, Kallitsaris A, Pournaras S, Messinis IE. The effect of estrogens on plasma ghrelin concentrations in women. J Endocrinol Invest. 2010;33(2):109-112. doi:10.1007/bf03346563

31. St-Pierre DH, Bastard JP, Coderre L, et al. Association of acylated ghrelin profiles with chronic inflammatory markers in overweight and obese postmenopausal women: a MONET study. Eur J Endocrinol. 2007;157(4):419-426. doi:10.1530/eje-07-0038

32. Ikezaki A, Hosoda $\mathrm{H}$, Ito $\mathrm{K}$, et al. Fasting plasma ghrelin levels are negatively correlated with insulin resistance and PAI-1, but not with leptin, in obese children and adolescents. Diabetes. 2002;51(12):3408-3411. doi:10.2337/ diabetes.51.12.3408
33. Sondergaard E, Gormsen LC, Nellemann B, Vestergaard ET, Christiansen JS, Nielsen S. Visceral fat mass is a strong predictor of circulating ghrelin levels in premenopausal women. Eur J Endocrinol. 2009;160(3):375-379. doi:10.1530/eje-08-0735

34. Zhang G, Yin X, Qi Y, et al. Ghrelin and cardiovascular diseases. Curr Cardiol Rev. 2010;6(1):62-70. doi:10.2174/157340310790231662

35. Li WG, Gavrila D, Liu X, et al. Ghrelin inhibits proinflammatory responses and nuclear factor-kappaB activation in human endothelial cells. Circulation. 2004;109(18):22212226. doi:10.1161/01.cir.0000127956.43874.f2

36. Dixit VD, Schaffer EM, Pyle RS, et al. Ghrelin inhibits leptin- and activation-induced proinflammatory cytokine expression by human monocytes and T cells. J Clin Invest. 2004;114(1):57-66. doi:10.1172/jci21134

37. Yano $\mathrm{Y}$, Toshinai $\mathrm{K}$, Inokuchi $\mathrm{T}$, et al. Plasma desacyl ghrelin, but not plasma HMW adiponectin, is a useful cardiometabolic marker for predicting atherosclerosis in elderly hypertensive patients. Atherosclerosis. 2009;204(2):590-594. doi:10.1016/j. atherosclerosis.2008.10.013

38. Kantorova E, Chomova M, Kurca E, et al. Leptin, adiponectin and ghrelin, new potential mediators of ischemic stroke. Neuro Endocrinol Lett. 2011;32(5):716-721.

39. Karim R, Stanczyk FZ, Brinton RD, Rettberg J, Hodis HN, Mack WJ. Association of endogenous sex hormones with adipokines and ghrelin in postmenopausal women. J Clin Endocrinol Metab. 2015;100(2):508-515. doi:10.1210/ jc.2014-1839

40. Isomoto $\mathrm{H}$, Ueno $\mathrm{H}$, Nishi $\mathrm{Y}$, et al. Circulating ghrelin levels in patients with various upper gastrointestinal diseases. Dig Dis Sci. 2005;50(5):833-838. doi:10.1007/s10620-0052648-z

41. Delporte C. Structure and physiological actions of ghrelin. Scientifica (Cairo). 2013;2013:518909. doi:10.1155/2013/518909

42. Meier U, Gressner AM. Endocrine regulation of energy metabolism: review of pathobiochemical and clinical chemical aspects of leptin, ghrelin, adiponectin, and resistin. Clin Chem. 2004;50(9):1511-1525. doi:10.1373/ clinchem.2004.032482

43. Michalski B, Krzemińska-Pakuła M, Kasprzak JD. [The way to the heart is through the stomach--the role of ghrelin in pathogenesis of cardiovascular disease]. Kardiol Pol. 2008;66(5):564-568.

44. Grinspoon S, Miller KK, Herzog DB, Grieco KA, Klibanski A. Effects of estrogen and recombinant human insulin-like growth factor-I on ghrelin secretion in severe undernutrition. J Clin Endocrinol Metab. 2004;89(8):39883993. doi:10.1210/jc.2004-0287

45. Lambrinoudaki IV, Christodoulakos GE, Economou EV, et al. Circulating leptin and ghrelin are differentially influenced by estrogen/progestin therapy and raloxifene. Maturitas. 2008;59(1):62-71. doi:10.1016/j.maturitas.2007.10.003

46. Steiger A. Ghrelin and sleep-wake regulation. Am J Physiol Regul Integr Comp Physiol. 2007;292(1):R573-574. doi:10.1152/ajpregu.00618.2006

47. Chen L, Xing T, Wang M, et al. Local infusion of ghrelin enhanced hippocampal synaptic plasticity and spatial 
memory through activation of phosphoinositide 3-kinase in the dentate gyrus of adult rats. Eur J Neurosci. 2011;33(2):266-275. doi:10.1111/j.1460-9568.2010.07491.x

48. Stoyanova II. Ghrelin: a link between ageing, metabolism and neurodegenerative disorders. Neurobiol Dis. 2014;72 Pt A:72-83. doi:10.1016/j.nbd.2014.08.026

49. Sowers MR, Wildman RP, Mancuso P, et al. Change in adipocytokines and ghrelin with menopause. Maturitas. 2008;59(2):149-157. doi:10.1016/j.maturitas.2007.12.006

50. Abdalla MM. Central and peripheral control of food intake.
Endocr Regul. 2017;51(1):52-70. doi:10.1515/enr-20170006

51. Loper HB, La Sala M, Dotson C, Steinle N. Taste perception, associated hormonal modulation, and nutrient intake. Nutr Rev. 2015;73(2):83-91. doi:10.1093/nutrit/nuu009

52. Uygun B, Kiyici S, Ozmen S, Gul Z, Sigirli D, Cavun S. The association between olfaction and taste functions with serum ghrelin and leptin levels in obese women. Endocrine Abstracts. 2017; 49 GP161. doi: 10.1530/endoabs.49.GP161

(C) 2020 The Author (s); This is an open-access article distributed under the terms of the Creative Commons Attribution License (http://creativecommons.org/licenses/by/4.0), which permits unrestricted use, distribution, and reproduction in any medium, provided the original work is properly cited. 